\title{
A qualitative exploration of 14 to 17-year old adolescents' views of early and preventative mental health support in schools
}

Mr Liam Spencer*, Research Assistant

Dr Ruth McGovern, Lecturer in Public Health Research

Professor Eileen Kaner, Professor of Public Health \& Primary Care Research

Population Health Sciences Institute

Newcastle University

Baddiley-Clark Building

Richardson Road

Newcastle upon Tyne

Tyne and Wear

NE2 4AX

UK

*Address correspondence to Liam Spencer, e-mail liam.spencer1@newcastle.ac.uk 


\begin{abstract}
Background: Preventative interventions may be effective in reducing and preventing symptoms of mental ill health in children and young people. However, there is a paucity of research in this area that explores the views of young people. This paper reports on a qualitative study, to inform the future development of attractive and appropriate early and preventative school-based mental health interventions.
\end{abstract}

Methods: Semi-structured interviews were conducted with a purposive sample of 12 young people aged 14 to 17 in North East England. Interviews were audio-recorded, transcribed, anonymised, and analysed following a thematic approach.

Results: Four key themes were identified, relevant to those providing, designing and commissioning early and preventative mental health interventions in schools: 'mental health literacy', 'risk factors for wellbeing decline', 'experience of school-based support', and 'recommendations for future support'.

Conclusions: Young people have varying levels of mental health literacy, but are able to identify academic stress, bullying, and the transition from primary to secondary school as leading causes of worry. Young people want more regular and in-depth mental health education, tailored levels of support in school, and improved training for teachers.

Keywords: public health, mental health, young people, early intervention, prevention, schools 


\section{Background}

Mental health problems constitute an increasing amount of the burden of disease worldwide ${ }^{1}$, and their prevention, and the provision of suitable mental health care are important public health issues ${ }^{2,3}$. In England, it is estimated that 1 in 8 young people (12.8\%) aged between 5 and 19 years are living with a diagnosable mental health disorder ${ }^{4}$, with $50 \%$ of mental health disorders presenting by the age of $14^{5}$, increasing to $75 \%$ by $18^{6}$. Despite this, there is evidence of a disparity between the prevalence of these disorders, and the proportion of young people accessing treatment, with as much as $70 \%$ of young people who experience mental health difficulties, not receiving appropriate interventions at a sufficiently early stage ${ }^{5,7}$.

Preventative approaches have been effective in reducing and preventing symptoms of mental ill health in children and young people ${ }^{8,9}$, and early identification and intervention greatly increases a young person's chances of being able to enjoy a healthy and productive future $^{10}$. Research suggests that school is the focal setting for young people to achieve positive mental health and emotional wellbeing, since schools are; the location where young people spend much of their time; easily accessible to families; and able to form community links ${ }^{11,12}$, with existing work showing promise for school-based mindfulness, and universal anxiety interventions ${ }^{8,9,13,14}$. The transition to secondary school presents a window of opportunity for developing interventions, aimed at improving both psychological functioning and educational attainment ${ }^{15}$. The Royal College of Psychiatrists have recognised that further development of early and preventative strategies is required ${ }^{5}$, with more needing to be done in order to provide guidance and support into schools ${ }^{16,17}$.

Mental health literacy refers to an understanding of how to obtain and maintain positive mental health; understand mental health issues and their treatments; decrease mental health stigma; and enhance help-seeking efficacy ${ }^{3,18}$. In 2018, the UK Government announced that mental health education would become a mandatory part of the curriculum for all schools from Autumn 202019, 20 . Previous work has emphasised the complex nature of young people people's mental health ${ }^{21}$, therefore providing appropriate and sustainable early support in schools can be difficult, and students have little say in this process ${ }^{11,14,16}$. It is vital that research actively seeks the views of young people ${ }^{22,23}$ so that effective and acceptable prevention strategies can be developed, and implemented ${ }^{24,25}$. Following the COVID-19 pandemic, it is also important to consider the impact of disrupted school routines, prolonged school closures, and strict social distancing measures on young people's mental health ${ }^{26}$ 
This paper builds upon the small body of existing qualitative literature, to provide further insight into young people's mental health, and their perspectives on early and preventative school-based support ${ }^{22,24}$. The aim of this research was to explore young people's lived experience of emotional and psychological challenges, which can negatively impact upon their mental health, in order to better inform the future development of school-based mental health support.

\section{Method}

\section{Design}

An exploratory qualitative study, using face-to-face semi-structured interviews with young people in the North East of England.

\section{Recruitment and Sample}

A selective purposive sampling method was used to recruit young people from a variety of backgrounds and localities, with national data on indices of multiple deprivation (IMD) ${ }^{27}$ used to identify schools located in diverse sociodemographic areas. Specific inclusion criteria for participants were: (1) to be aged between 14 and 17 years old; (2) in mainstream local authority education; and (3) parental and participant consent to participate obtained. The lower age of 14 was set as this marks the start of Key Stage 4, which has previously been identified as a significant source of stress and worry ${ }^{28}$. The upper age of 17 was set, as from 18 years of age young people are not required to remain in education ${ }^{29}$, and would be eligible to access adult mental health services ${ }^{30}$. An exclusion criterion was imposed on participants receiving treatment for pre-existing mental health issues, as the focus of this research was on early intervention and prevention. Recruitment was conducted via contact with secondary school staff and youth workers in the North East of England, who acted as gatekeepers, and were encouraged not to approach potential participants directly, but to share information about the study with groups, and ask interested individuals to approach them for more information.

\section{Data collection}

A semi-structured interview schedule was developed (Appendix 1), which was designed to guide topics of interest covered in the interviews. As part of the data collection, vignettes (Appendix 2) representing different levels of school support; universal, selective and indicated $^{8}$, were used to encourage participants to consider scenarios that mimic reality ${ }^{31}$, and obtain their perceptions, opinions, beliefs and attitudes in a less intrusive or threatening way $^{32,33}$. A workshop with a representative local youth group was conducted to refine 
interview materials, and ensure they were appropriate. Young people who expressed an interest in participating were provided with an information leaflet prior to providing consent and taking part in the interview. Participants were not provided with a financial incentive, as this may have unduly influenced young people's decision to participate ${ }^{34}$. Data were collected by one researcher (LS) within schools and other youth/community facilities, and data collection ceased when no new information was attained ${ }^{35}$. The interviews were audio recorded and transcribed verbatim by a professional transcription company, to facilitate quicker progression of the data analysis process. The transcripts were fully anonymised, with the names of people and places omitted, to protect their anonymity.

\section{Analysis}

A thematic method, following the guidelines set out by Braun and Clarke ${ }^{36}$ was used to analyse the transcribed data. This bottom-up inductive approach, driven by the data, was chosen as an accessible and theoretically flexible approach to analysing qualitative data ${ }^{36}$. A small number of a priori codes were defined considering the stated aims of the project, and refined through drawing on key issues present within existing research. Emergent themes were discussed amongst the researchers, and NVivo 11 was used in the organisation and analysis of data. Original codes and sub-theme categories were organised into four key themes, identified as relevant areas of focus for those providing, designing, and commissioning early and preventative mental health interventions in schools.

\section{Findings}

\section{Sample}

Twelve young people aged 14 to 17 years old participated in the study. The median age of participants was 15 years $(S D=1.07)$. Six participants identified as female, and six identified as male. All participants identified as White British. All schools were state funded, operating within and outside (academy status) local authority control. Each school provided differing levels of mental health support, and none were part of the NHS Trailblazer programme ${ }^{37}$. IMD levels for the Lower Layer Super Output Areas (LSOAs) corresponding to the schools' catchment areas were as follows: 'high level of deprivation' (3 schools; 4 participants); 'medium level of deprivation' (2 schools; 2 participants); and 'low level of deprivation' (3 schools; 6 participants $)^{27}$.

The four key themes were: mental health literacy, risk factors for wellbeing decline, experiences of school-based support, and recommendations for future support. These themes are described below and illustrated using verbatim participant quotations. 


\section{Mental health literacy}

Participants demonstrated varying levels of mental health literacy, with some able to articulate what they felt it meant to have 'good mental health'. Participants described circumstances and events with positive or negative impacts on their mental health and identified protective factors that contributed to positive emotional wellbeing.

"A positive outlook on things, you're obviously not distressed or anything. There's nothing constantly occupying your mind, like the same thing, and you feel able to cope with things and stuff. Obviously saying something is wrong with your mental health, that's quite serious. But then if you have mental health in general, it doesn't have to be a bad thing." (Female, aged 17)

Participants described the expectation that young people should understand mental health issues, regardless of any relevant mental health education they may or may not have received in school. Some felt the burden of responsibility to gain relevant information was theirs, in order to develop the ability to understand and process their own emotions.

"I think it's not discussed, because I think everybody knows it. Sometimes we do get told about it, and you always get one, but it's not one of the main topics." (Male, aged 15)

"Everyone's obviously got mental health, but not everyone realises that, and then it's quite negative the way people talk about it." (Male, aged 17)

The two main mental health issues that participants described were depression and anxiety, although they recognised that self-harm and suicide were often linked to these disorders. Despite evidence of some awareness and understanding, the general sense from participants was that information derived from school was not sufficiently in-depth. Some participants showed deeper understanding and described how others often misunderstood these issues or did not fully understand their complexity and individuality.

"You'll hear, "Depression is sadness." It's more than that, it's a lot more than that. A lot of people don't understand." (Female, aged 15)

\section{Risk factors for wellbeing decline}

Participants identified a range of risk factors, which they felt could cause young people to be vulnerable to a decline in mental health and emotional wellbeing. Key risks were the transition from primary to secondary school, bullying, and increasing academic pressures. 
The transition from primary to secondary school represented a significant milestone and source of stress in young people's lives. The transition to a much larger school site and mixing with a lot of new people could increase the pressure to conform; with young people concerned about not fitting in, and the possibility of being judged.

"I think it's meeting new friends and, obviously, going to a new school, it's quite stressful and you don't know if you'll fit in. You don't know if you'll get judged, and that." (Female, aged 14)

A recurrent theme was bullying, and participants spoke about experiencing discrimination, and an absence of kindness from peers. As well as being a potential trigger for mental health issues, one participant referred to bullying being used to target those who had openly discussed their experience of mental ill-health, and suggested that this behaviour may be driven by individuals' own wellbeing issues.

"I think bullying comes from a lot of reasons. It could come from a lack of understanding. It could come from the fact they've got issues themselves." (Male, aged 17)

Many participants highlighted the academic pressures of school life. Young people described dealing with multiple challenges in their lives alongside with large amounts of homework, and upcoming exams were frequently described as causing excessive stress. Participants emphasised how this feeling of pressure increased as young people moved up through the school years and that feelings of overwhelming stress caused by the pressure to prepare for examinations could lead to isolation.

"Like sometimes, starting GCSEs from Year Nine and then building it up through the years, a lot of homework is put onto us. Sometimes, it's due in for the next day and it's a massive load. So, I think stress makes everything worse as well." (Female, aged 15)

"Near our exam time, most of my friends were quite sad and depressed because they felt like they were just sitting in the house and not really doing much and they were just consumed by all this exam stress, that they couldn't do anything." (Male, aged 16)

\section{Experiences of school-based support}

Participants had some experience of receiving learning-based sessions in school, designed to increase mental health literacy and reduce stigma. Participants spoke about attending assemblies regarding mental health, but largely reported not feeling well-informed following these sessions. Some schools were more actively engaged in regularly promoting positive 
mental health and wellbeing in this format; however, the delivery of such sessions was often short-term and initiated in anticipation of an exam period

“There was a week when we did mental health awareness, but I don't think they went into how much more serious it actually is than what they should've done." (Female, aged 15)

"I think it might have been discussed in Year 7, but other years, you have a weekly assembly normally, and I think we've had it discussed once or twice in there." (Female, aged 16)

Some participants had been signposted by teachers to mental health support, but examples that they provided suggested this was not actively promoted by staff. At times it could seem as if problems were being passed on for others to address. Participants spoke about having positive relationships with some teachers and feeling comfortable approaching them for support. Despite not being mental health practitioners, participants felt that certain teachers, with whom they shared a positive and trusting relationship, could often provide sufficient early support by listening to and legitimising their feelings.

"I think some teachers, if you go to them and you say, "I think l've got mental health," they say, "Well, speak to this person"." (Male, aged 16)

"My form tutor knows more stuff, things, that's happening, because I was really close to him all the way through school. He knows my past, so I feel comfortable talking to him about it." (Female, aged 15)

Conversely, some participants spoke about how it could be difficult to speak about their emotional or psychological concerns, and that many teachers did not have an adequate understanding of mental health issues, which could discourage disclosure.

"The majority of them do not understand mental health themselves, so don't understand how to help those with mental health problems." (Male, aged 17)

\section{Recommendations for future support}

Participants reported that there should be an increase in the frequency and scope of mental health education in schools. It was suggested that sessions should be more detailed, advocating a regular combination of assemblies, and smaller classroom-based activity. Participants also felt it was important that the availability of support should be better advertised, therefore normalising help-seeking. This approach could also encourage teachers to be more proactive in identifying problems and helping student's access support. 
"I hope that the information on mental health does change. It should be on, "What is it? How do you treat it? How serious it is." For example, interviews with people suffering from depression." (Male, aged 16)

"If someone notices something, then a teacher can approach them. Not force them into, because that would cause stress, but just let them know, "You've got support here. If you want it, it's yours"." (Female, aged 15)

Participants expressed a desire for a physical space in schools, where young people could go if they were struggling with their emotions; to relax, be in private, and feel safe.

Participants felt this would create an opportunity for staff to approach them and offer support. Participants also spoke of how they viewed teachers not only as providers of education, but as sources of emotional and practical support. They expressed the need for school staff to receive better mental health training, to have a good understanding of the issues that can impact upon young people, and how they may better support students.

"I think it would be nice for everyone to know that there is somewhere they can go.

Somewhere in school, which is private, and doesn't look very plain. Somewhere which has colour, looks comfortable, so I can feel relaxed." (Female, aged 15)

"I feel like funding could be spent on training teachers and educating teachers on more mental health issues." (Female, aged 17)

Participants felt it was important that support should be offered to young people at an early stage, before the development of potentially serious mental health issues. They identified how this support could be offered during the transition to secondary school, and that it should be increasingly provided as the academic pressures on young people grow.

"Maybe a brief talk in primary school, but as soon as you hit secondary school there would be more of a deep conversation about it." (Female, aged 14)

"As soon as you get to secondary school, it becomes a lot more serious and stress can easily take over you." (Male, aged 15)

\section{Discussion}

\section{Main findings of this study}


This study revealed that young people: (i) are familiar with the concepts of anxiety and depression, but have varying levels of mental health literacy; (ii) identify bullying, academic pressure, and the transition from primary to secondary school as key causes of worry; (iii) have mixed experiences of receiving school-based mental health support, emphasising the importance of the quality of the relationship between themselves and school staff; and (iv) highlighted the necessity of more frequent and in-depth delivery of mental health education, tailored levels of support, improved signposting to support, safe spaces in schools where they can feel relaxed and comfortable discussing sensitive issues, and better mental health training for school staff.

\section{What is already known on this topic?}

Early and preventative approaches have been effective in reducing and preventing symptoms of anxiety and depression in children and young people $\mathrm{e}^{8-10,13,14}$, and schools are a focal setting for young people to achieve positive mental health and emotional wellbeing ${ }^{11}$, 12. Previous work has identified that early and preventative approaches are; the least intrusive, are usually of low cost, and do not exclude any young people who might benefit from what is offered, and therefore have the greatest chance of being implemented in schools $^{38}$. It has been recognised that further development of early and preventative strategies is required, which actively seeks the views of young people ${ }^{11,22-24}$, and that more needs to be done in order to provide guidance and support into schools $5,16,17,25$.

\section{What this study adds}

This study has given voice to young people in North East England regarding emotional and psychological challenges, and provided an opportunity for researchers to undertake a journey of learning and questioning of personal beliefs, whilst portraying important aspects of social phenomena within the context of those experiencing them ${ }^{39,40}$. There is a need for improved training provision for school staff, to facilitate young people's desire for more regular and in-depth mental health education and support. Young people would benefit from flexible and personalised support in school, and an environment that facilitates positive conversations about mental health, encourages help-seeking, and works harder to identify when young people may be struggling. Schools must be acutely aware of the impacts of bullying, academic stress, and the transition from primary school to secondary school. Future policies and support should reflect this, and should be co-developed with young people ${ }^{41}$. Young people were able to weigh up the strengths and limitations of school-based mental health interventions, and the feasibility and acceptability of preventative school-based mental health support must be examined further. The study sample was socioeconomically diverse, and had an equal male-female ratio, which was controlled for through recruitment, 
to make the small sample as representative of the region as possible. Whilst not much is known about the long-term mental health effects of large-scale disease outbreaks on young people, the long-term impacts of the COVID-19 pandemic should be considered in future research in this field ${ }^{26}$.

\section{Limitations of this study}

Recruitment relied on the assistance of gatekeepers and the data collection period was short. Hence our sample was relatively small, even by the standards of qualitative research work, therefore reflections on some schools were based on only one participant. Initial recruitment issues meant that data collection was undertaken during the summer term, therefore some potential participants were busy preparing for exams. As such, the median age of participants was 15, and future work should aim to increase participation from older adolescents. Undertaking a small number of interviews can facilitate a close association with interview participants, enhancing the rigour of in-depth enquiry ${ }^{42}$, through a considered approach to the complexity and context of individual participants' experiences ${ }^{43,44}$. The average length of interviews in the present study was short at approximately 30 minutes, however succinct responses are typical in qualitative work with young people ${ }^{41}$. The generation of empirical data with a relatively narrow focus is possible through short interviews ${ }^{45}$, which can glean interesting data due to the co-operative nature of interview participants ${ }^{46}$. Due to the research focus on early intervention and prevention, the inclusion criteria specified that participants should not have a mental health diagnosis, however future research may benefit from exploring the findings presented here with a diagnosed population. Only one method of data collection was used; conducting focus groups may have generated greater breadth of data, due to the interactions and conversations that could have been facilitated amongst peers in a group setting. Nevertheless, due to the sensitive nature of the topic, one-to-one interviews were deemed more appropriate, to gently probe participants and achieve a sufficient depth of understanding of what can be difficult topics to discuss. Recruitment gatekeepers may have identified participants they thought would be best suited to taking part in the study, and thus this may have excluded some less engaged or less vocal young people. Participants' knowledge or interest in the topic may also have increased their motivation to participate.

\section{Conclusion}

To suit the expressed needs of mid-adolescents, mental health support provided in schools should be more regular and wider in scope than currently provided. There is also a need for improved training for school staff, flexible and personalised levels of support in school, and an environment that facilitates positive conversations about mental well-being. Schools need 
to encourage help-seeking and proactively work to identify when young people may be struggling. Further intervention development work is required with young people to coproduce what acceptable, effective school-based mental health support looks like.

\section{Acknowledgements}

Ethical approval was granted by Newcastle University (Faculty of Medical Sciences) Ethics Committee (Ref: 1462/2836) on 30th January 2018. We would like to thank all participants for giving up their time to take part in this study, and for sharing their views and experiences.

Liam Spencer drafted the original manuscript, with the co-authors contributing to the article development and approved final version. 


\section{References}

1. Ferrari A, Charlson F, Norman R, Patten S, Freedman G, et al. Burden of depressive disorders by country, sex, age, and year: findings from the global burden of disease study 2010. PLoS medicine. 2013;10(11).

2. Reijneveld S. Mental health as a public health issue. European Journal of Public Health. 2005;15(2):111.

3. Vidourek R, Burbage M. Positive mental health and mental health stigma: A qualitative study assessing student attitudes. Mental Health \& Prevention. 2019;13:1-6.

4. Mental Health of Children and Young People in England, 2017. NHS Digital; 2018.

5. No health without public mental health: The case for action. Royal College of Psychiatrists London; 2010.

6. Kim-Cohen J, Caspi A, Moffitt T, Harrington H, Milne B. Prior juvenile diagnoses in adults with mental disorder: developmental follow-back of a prospective-longitudinal cohort.

Archives of general psychiatry. 2003;60(7):709-17.

7. Future in mind: Promoting, protecting and improving our children and young people's mental health and wellbeing. Department of Health; 2015.

8. Neil A, Christensen $\mathrm{H}$. Efficacy and effectiveness of school-based prevention and early intervention programs for anxiety. Clin Psychol Rev. 2009;29(3):208-15.

9. Feldner M, Zvolensky M, Schmidt N. Prevention of Anxiety Psychopathology: A Critical Review of the Empirical Literature. Clinical Psychology: Science and Practice.

2004;11(4):405-24.

10. Making Life Better: A Whole System Strategic Framework for Public Health 2013-2023. Department of Health Social Services and Public Safety, Belfast; 2014.

11. Churchman A, Mansell W, Al-Nufoury Y, Tai S. A qualitative analysis of young people's experiences of receiving a novel, client-led, psychological therapy in school. Counselling and Psychotherapy Research. 2019;19(4):409-18.

12. Carta M, Di Fiandra T, Rampazzo L, Contu P, Preti A. An Overview of International Literature on School Interventions to Promote Mental Health and Well-being in Children and Adolescents. Clinical Practice \& Epidemiology in Mental Health. 2015;11(Suppl 1):16-20.

13. Sapthiang S, Van Gordon W, Shonin E. Health School-based Mindfulness Interventions for Improving Mental Health: A Systematic Review and Thematic Synthesis of Qualitative Studies. Journal of Child and Family Studies. 2019:1-9.

14. Skryabina E, Morris J, Byrne D, Harkin N, Rook S, et al. Child, teacher and parent perceptions of the FRIENDS classroom-based universal anxiety prevention programme: A qualitative study. School mental health. 2016;8(4):486-98.

15. Riglin L, Frederickson N, Shelton K, Rice F. A longitudinal study of psychological functioning and academic attainment at the transition to secondary school. Journal of adolescence. 2013;36(3):507-17. 
16. Phillippo K, Kelly M. On the fault line: A qualitative exploration of high school teachers' involvement with student mental health issues. School Mental Health. 2014;6(3):184-200.

17. Newham J, Henderson E, McGovern R, Russell S, Spencer L, et al. PROMOTE: NE Preventing Risks Of Mental illness Onset and Treating Early in the North East of England. Newcastle: Fuse: The Centre for Translational Research in Public Health; 2017.

18. Kutcher S, Wei $Y$, Coniglio $C$. Mental health literacy: past, present, and future. The Canadian Journal of Psychiatry. 2016;61(3):154-8.

19. Long R. Relationships and sex education in schools (England). 2019.

20. Relationships and Sex Education (RSE) and Health Education: Draft statutory guidance for governing bodies, proprietors, head teachers, principals, senior leadership teams, teachers. London: Department for Education; 2019.

21. Cairns K, Yap M, Rossetto A, Pilkington P, Jorm A. Exploring adolescents' causal beliefs about depression: A qualitative study with implications for prevention. Mental Health \& Prevention. 2018;12:55-61.

22. Plaistow J, Masson K, Koch D, Wilson J, Stark R, et al. Young people's views of UK mental health services. Early intervention in psychiatry. 2014;8(1):12-23.

23. Burns J, Rapee R. Adolescent mental health literacy: young people's knowledge of depression and help seeking. Journal of adolescence. 2006;29(2):225-39.

24. Derges J, Kidger J, Fox F, Campbell R, Kaner E, et al. Alcohol screening and brief interventions for adults and young people in health and community-based settings: a qualitative systematic literature review. BMC public health. 2017;17(1):562.

25. Sheffield J, Spence S, Rapee R, Kowalenko N, Wignall A, et al. Evaluation of universal, indicated, and combined cognitive-behavioral approaches to the prevention of depression among adolescents. Journal of consulting and clinical psychology. 2006;74(1):66.

26. Lee J. Mental health effects of school closures during COVID-19. The Lancet Child \& Adolescent Health. 2020;4(6):421.

27. McLennan D, Noble S, Noble M, Plunkett E, Wright G, et al. The English Indices of Deprivation 2019: technical report. 2019.

28. Putwain D. Assessment and examination stress in Key Stage 4. British Educational Research Journal. 2009;35(3):391-411.

29. Lambert S, Maylor U, Coughlin A. Raising of the participation age in the UK: The dichotomy between full participation and institutional accountability. 2015.

30. Ubido J, Scott-Samuel A. Rapid evidence review series: effective pathway from child to adult mental health services. Liverpool Public Health Observatory, Liverpool. 2015.

31. Törrönen J. Using vignettes in qualitative interviews as clues, microcosms or provokers. Qualitative Research Journal. 2018.

32. Morrow V. Using qualitative methods to elicit young people's perspectives on their environments: some ideas for community health initiatives. Health education research. $2001 ; 16(3): 255-68$. 
33. Azman $\mathrm{H}$, Mahadhir M. Application of the vignette technique in a qualitative paradigm. GEMA Online Journal Of Language Studies. 2017;17(4).

34. Faden R, Beauchamp T. A history and theory of informed consent: Oxford University Press; 1986.

35. Wuest J. Are we there yet? Positioning qualitative research differently. Qualitative Health Research. 2011;21(7):875-83.

36. Braun V, Clarke V. Using thematic analysis in psychology. Qualitative Research in Psychology. 2006;3(2):77-101.

37. Cortina M, Shipman J, Saunders F, Day L, Blades R, et al. Embedding interagency working between schools and mental health specialists: A service evaluation of the Mental Health Services and Schools and Colleges Link Programme workshops. Clinical child psychology and psychiatry. 2019;24(4):906-20.

38. Stallard P. Mental health prevention in UK classrooms: the FRIENDS anxiety prevention programme. Emotional and Behavioural Difficulties. 2010;15(1):23-35.

39. Corlett S, Mavin S. Reflexivity and researcher positionality. The SAGE handbook of qualitative business and management research methods. 2018:377-99.

40. Palaganas E, Sanchez M, Molintas V, Caricativo R. Reflexivity in qualitative research: A journey of learning. Qualitative Report. 2017;22(2).

41. Lynch E, McGovern R, Elzerbi C, Breckons M, Deluca P, et al. Adolescent perspectives about their participation in alcohol intervention research in emergency care: A qualitative exploration using ethical principles as an analytical framework. PloS one. 2019;14(6).

42. Crouch $M$, McKenzie $H$. The logic of small samples in interview-based qualitative research. Social science information. 2006;45(4):483-99.

43. Beeson D. Nuance, complexity, and context: qualitative methods in genetic counseling research. Journal of genetic counseling. 1997;6(1):21-43.

44. Vasileiou K, Barnett J, Thorpe S, Young T. Characterising and justifying sample size sufficiency in interview-based studies: systematic analysis of qualitative health research over a 15-year period. BMC medical research methodology. 2018;18(1):148.

45. Tjora A. Qualitative research as stepwise-deductive induction: Routledge; 2018.

46. Bryman A. Chapter 15: Interviewing in qualitative research. Social research methods. $2004 ; 2$. 


\section{Appendix 1}

Thank you for taking part in my project. I would like to ask you some questions about yourself, things that you think may impact on young people's health and happiness, and your thoughts about the best way that schools/colleges can help to improve this.

- Reiterate issues of confidentiality and anonymity

- The purpose of the study

- What is going to happen with the data

- Complete consent forms

\section{1) Introduction}

- Would you like to tell me a little bit about yourself before we get started?

- Prompts: age, school/college, family, hobbies, interests

\section{2) Mental health literacy}

- When people talk about 'mental health', what terms are used?

- Is mental health talked about at school/college?

- Do teachers discuss or teach about mental health?

- Is it something friends chat about? (Positive or negative)

- Do you know anyone who has received support for mental health issues?

- What kind of issues do your friends talk about experiencing?

- Prompts: anxiety, panic, low-mood, depression, stress, worry

- Emphasise confidentiality and disclosures

- How is mental health portrayed through social media?

\section{3) Levels of intervention}

- Show scenarios, which relate to different levels of intervention. Use post-it notes to highlight the strengths and weaknesses of each example.

\section{4) Ideal intervention}

- If we were going to offer a new way of supporting young people's mental health and wellbeing in school, what would you like it to look like? Prompts:

- What ages?

- Any particular young people, or everyone?

- Who would be delivering intervention?

- What size group, or one-to-one?

- Who would be informed about a young person being involved?

- Would young people need an incentive for participating?

\section{6) Close}

- Offer the participants the opportunity to share or add anything else they may feel is important or relevant.

Thank the participant, and offer reassurance that all of the responses will be anonymised, that they will not be identified in the dissemination of results, and if they wish they will be able to receive information about the findings. Offer participants a copy of the local services signposting sheet. 


\section{Scenario 1}

- This teacher is delivering a session to her form class about mental health as part of the school timetable.

- The session includes general information about what is meant by 'mental health'.

- Everyone is included, whether or not they have experienced any mental health issues.

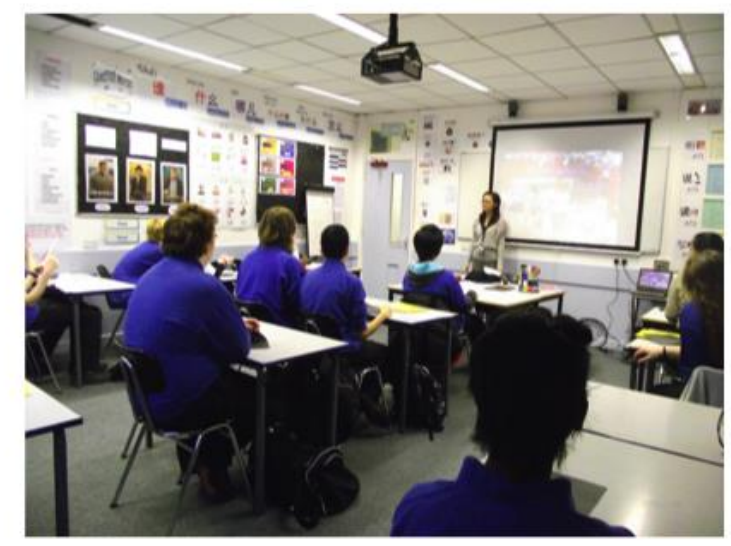

\section{Scenario 2}

- This small group of young people have been invited to take part in a session at lunch time, with a teacher.

- The session is about understanding feelings and managing behaviour.

- These young people may have been getting into trouble at school, or not doing very well with their work.

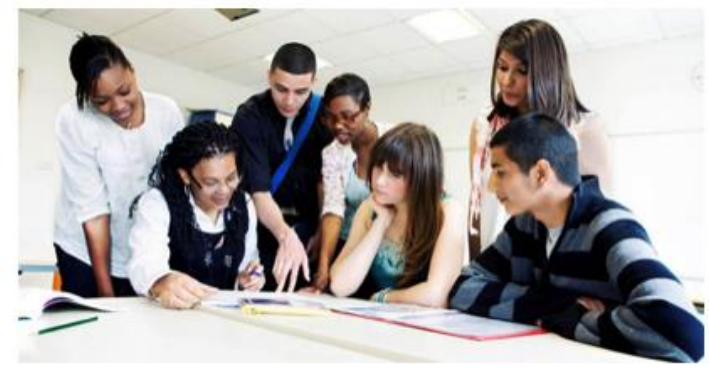




\section{Scenario 3}

- Jenny is meeting with a teacher at school who has received mental health training.

- She had been to her GP about depression, but her symptoms were not severe enough to be offered counselling.

- Jenny spoke to her form tutor about what had happened, and was told the school could offer her support to help improve her mood.

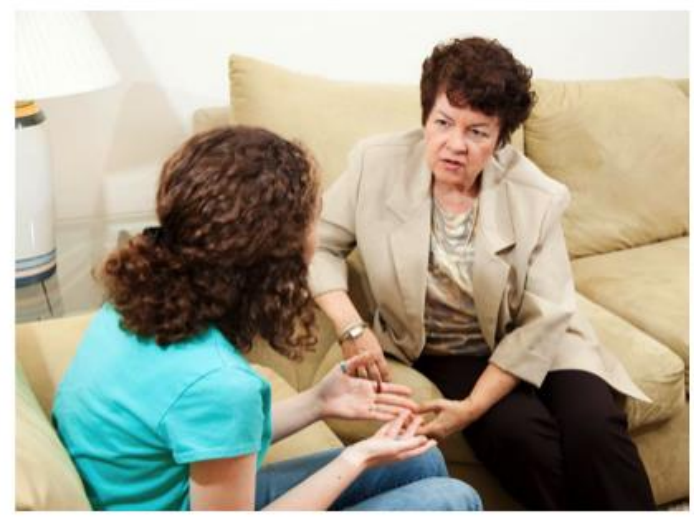

